Submission ID: 43728

\title{
Universal Algorithmic System for Geophysical Objects Appoximation Based
} on Fourier-Splines

\section{A.S. Gorshkov* (Lexion Development)}

\section{SUMMARY}

The universal algorithm of geophysical maps with faults synthesis for seismic objects is developed. The new method concludes the approximation of both regular and non-regular data with the new finite functions class called "Fourier-splines" having great advantages versus well-known approaches particularly cubical Bsplines.

The innovation of the new solution is $3 \mathrm{D}\{\mathrm{xyz}\}$ Fourier-splines arrays synthesis on the parametrical 2D \{uv\} grid aligned along the profiles grid. The fault surfaces may be built additionally on the corresponding local parts of profiles similarly to isolines in automatic mode.

The numerical algorithm is optimized with utilization of more efficient version of square-root method of linear systems resolution and implementation on the base of parallel processing technique NVIDIA CUDA. As a result we have unified multi-dimensional method of different objects synthesis in the best splines from the arbitrary non-regular data.

The new advanced solution has the next advantageous features versus the known ones:

- the unified approach to maps and faults synthesis providing arbitrary complex 3D models including maps of z-horizon type (with local negative trends);

- adapting the parametrical grid to profile grid providing to the most precise approximation;

- the absence of edge contouring (masking), softened map edges are formed intrinsically. 
Универсальный математический аппарат аппроксимации геофизических объектов на основе нового класса фукций «фурье-сплайны»

А.С. Горшков* (EAGE)

Введение

Материал настоящего доклада представляет обобщение результатов, которые были ранее представлены в докладах автора на «Геомодели-2008/2009/2016».

В основе метода аппроксимации как регулярных, так и нерегулярных данных включая сейсмические лежит предложенный автором новый класс финитных функций «фурьесплайны», имеющий принципиальные преимущества перед известными методами, например, традиционными кубическими В-сплайнами. Алгоритмически метод оптимизирован применением в решении для обращения системных общих и ленточных регрессионных матриц усовершенствованного метода квадратного корня. Программная реализация метода дополнительно ускорена применением технологии параллельных вычислений NVIDIA CUDA.

В итоге получен единый многомерный алгоритм синтеза объектов различной природы в сплайнах на основе произвольных как регулярных, так и нерегулярных данных.

Предложенное математическое решение отличают следующие преимущества перед известными:

- гибкое и эффективное сочетание всех классических математических функциональных базисов (алгебраические и тригонометрические полиномы, сплайн-конволюция), дополненное новым классом «фурье-сплайны» конволюционного типа;

- общий 3D подход к синтезу объектов (в частности, геофизическиз карт с разломами), позволяющий получать любые сложные комбинации 3D моделей, в том числе карты типа $z$ горизонтов (с отрицательным наклоном);

- эффективная адаптация в параметрических координатах, обеспечивающая наиболее точную аппроксимацию любых объектов.

\section{Содержание метода}

Исходные сейсмические данные можно классифицировать как числовые массивы различной размерности:

- 1D (отдельная сейсмотрасса),

- 2D (карта, геофизический «горизонт»),

- 3D (сейсмический «профиль»),

- 4D (сейсмический «куб»).

Сейсмические данные согласно новому методу могут быть обработаны посредством конволюции

$$
f(x)=\sum_{k=0}^{N-1} A(k) \varphi(x-k)
$$

на базисе специального класса финитных функций «Фурье-сплайны», построенных на основе рядов Фурье малого порядка, с общей формулой:

$$
\varphi_{\mathrm{N}}(x)=2^{2 \mathrm{~N}-1} \cos ^{2 \mathrm{~N}}\left(\frac{\pi x}{4}\right)
$$


При $N=2$ имеет место наиболее простой выбор, достаточный практически для большинства условий в силу непрерывности первой и второй производных:

$$
\varphi(x)=8 \cos ^{4}\left(\frac{\pi x}{4}\right)=3+4 \cos \left(\frac{\pi x}{2}\right)+\cos (\pi x)
$$

а также с использованием ортогонального дополнения (исключение постоянной компоненты и замена $\cos ->-\sin , \sin ->\cos$, что известно как «преобразование Гильберта»):

$$
\varphi^{*}(x)=-4 \sin \left(\frac{\pi x}{2}\right)-\sin (\pi x)
$$

Конволюционная аппроксимация достигается посредством решения обратной задачи декомпозиции разрешением соответствующей регрессионной системы линейных уравнений новыми методами, обобщающими как известные классические, так и так называемые специальные:

- последовательной ортогонализации базиса системы,

- параллельной ортогонализации базиса системы,

- квадратного корня для слабозаполненных мульти-ленточных матриц с адаптивной перестановкой строк/столбцов с целью снижения числа нулевых элементов,

- сопряженных градиентов для слабозаполненных мульти-ленточных матриц с наиболее

компактным представлением в памяти компьютера;

Посредством конволюции результата преобразования с данными сплайнфункциями далее возможно выполнить следующие операции:

- дифференцирование,

- интегрирование,

- интерполирование,

- преобразование в иные функции или функционалы (в частности: огибающая, фаза, частота),

- аппроксимацию решений обратных задач (например, преобразование координат, получение изолиний и изоповерхностей).

Классификация нового подхода среди известных классических может быть представлена следующей Таблицей 1.

\section{Таблица 1.}

\begin{tabular}{|c|c|c|}
\hline Полиномы & Алгебраические полиномы & $\begin{array}{c}\text { Тригонометрические } \\
\text { полиномы (ряды Фурье) }\end{array}$ \\
\hline$P_{N}(x)=\sum_{k=0}^{N-1} a(k) x^{k}$ & $P_{N}(x)$ & $P_{N}\left(e^{j x}\right)$ \\
\hline Сплайн-конволюция & В-сплайн $(N=0 \ldots 3)$ & $\varphi(x)=\cos ^{4}(\pi x / 4)$ \\
$f(x)=\sum_{k=0}^{N-1} a(k) \varphi(x-k)$ & & \\
\hline
\end{tabular}

Применение нового аппарата обработки данных позволяет в том числе выполнить кинематическую интерпретацию, включающую извлечение изолиний и изоповерхностей, а также динамическую интерпретацию:

- атрибутный анализ, 
- инверсию,

- сейсмофациальный анализ,

- подбор модели скоростей и преобразование время/глубина;

- построение структурных карт в глубинном масштабе и карт прогнозных параметров посредством:

- синтеза массивов 1D Фурье-сплайн коэффициентов, полученных в результате конволюционной аппроксимации, на регулярной 2D координатной $\{x y\}$-сетке;

- синтеза массивов 3D Фурье-сплайн коэффициентов, полученных в результате конволюционной аппроксимации, на 2D параметрической координатной $\{u v\}$-сетке, согласованной с профильной $\{x y\}$-сеткой;

- синтеза массивов 2D Фурье-сплайн коэффициентов преобразования профильной $\{x y\}-$ сетки в параметрическую координатную \{uv\}-сетку;

- многополосного синтеза вышеуказанных коэффициентов с получением массивов размерностью в $1 \mathrm{D}-4,8,16 \ldots$, в 2D - 4x4,8x8,16x16... и т.д. до требуемой детализации;

- выявление зон аномалий, соответствующих ловушкам нефти и газа, и прогнозирование петрофизические свойства месторождения.

Символически применение нового аппарата к данным различной размерности иллюстрируется Таблищей 2.

Таблица 2.

\begin{tabular}{|c|c|c|}
\hline Объект & Предобработка & Синтез \\
\hline $\begin{array}{c}\text { Сейсмотрасса: } \\
\text { 2D/3D }\end{array}$ & $S^{-1}$ & $S+S \& S^{*}$ \\
\hline Профиль: 3D & $S_{x}^{-1}\left|S_{t}^{-1}+P_{x}^{-1} \& S_{x}^{-1}\right| S_{t}^{-1}$ & $S_{x}\left|S_{t}+P_{x} \& S_{x}\right| S_{t}$ \\
\hline Куб: 4D & $S_{x}^{-1}\left|S_{y}^{-1}\right| S_{t}^{-1}+$ \\
\hline $\begin{array}{c}\text { Карта } \\
\text { (горизонт): 3D }\end{array}$ & $P_{x}^{-1} \& S_{x}^{-1}\left|P_{y}^{-1} \& S_{y}^{-1}\right| S_{t}^{-1}$ & $S_{x}\left|S_{y}\right| S_{t}+P_{x} \& S_{x}\left|P_{y} \& S_{y}\right| S_{t}$ \\
\hline
\end{tabular}

Вычислительная оптимизация в реальном времени достигается на основе технологий OpenGL GLSL и NVIDIA CUDA.

Разработанный математический аппарат и практическая технология цифровой обработки данных любой природы наряду с решением сейсмических задач (Рис.1,2) также были успешно применены в других отраслях - CAD/CAM/CAE, медицинская томография, распознавание образов, нейронные сети и др.

\section{Выводы}

Предложенный математический аппарат в итоге является особенно удачной основой для разработки новых цифровых стандартов представления, хранения и передачи цифровых данных (в том числе альтернативно широко распространенному стандарту jpeg/mpeg), а также систем цифрового телевидения высокой четкости (HDTV). 


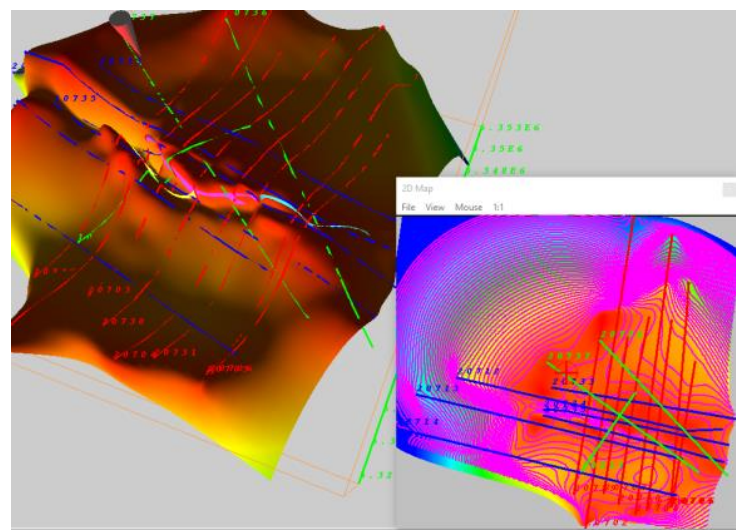

Рисунок 1. Синтезированная карта с нарушениями в изолиниях.

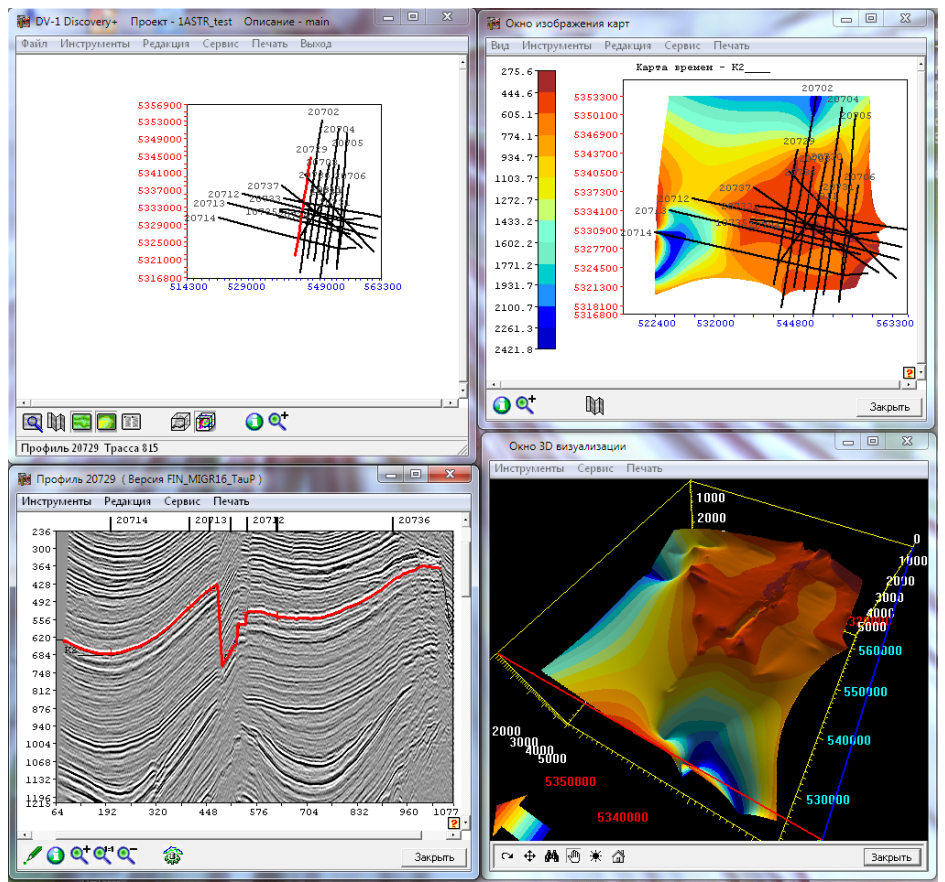

Рисунок 2. Применение новой технологии в прикладном пакете.

\section{Библиография}

1. Горшков А.С. Цифровая обработка сигналов: атомарные функции и теория чисел. Москва: Машиностроение., 1994 г. - 224 с.

2. Горшков А.С. Обобщенная система линейных алгоритмов для цифровой обработки информации. Доклады Академии наук СССР/Мат. физика.-1994.-Т 336.-N 5.-С. 29-32.

3. Горшков A.С. Пакет алгоритмов компьютерной 3D-графики для CAD-CAMприложений. САПР и графика. N 3. 2003 г.

4. Универсальный метод обработки и визуализации сейсмического куба данных на основе фурье-сплайн декомпозиции. / Горшков А.С. // Тезисы к докладу на конф. «Геомодель2008».-22-25/9-2008

5. Универсальная система синтеза изоповерхностей сейсмического куба. / Горшков А.С. // Тезисы к докладу на конф. «Геомодель-2009».-7-10/9-2009.

6. Универсальный алгоритм параметрического синтеза геофизических карт с нарушениями на основе фурье-сплайнов. / Горшков А.С. // Тезисы к докладу на конф. «Геомодель-2016».-11-15/9-2016. 


\section{References}

1. Gorshkov A.S. Digital Signal Processing: Atomic Functions and Number Theory. Moscow: Mashinostroeniye., 1994. - 224 pp.

2. Gorshkov A.S. The Generalized Linear Algorithms System for Digital Information Processing. Doklady Akademii Nauk SSSR//Math. Physics.-1994.-V 336.-N 5.-PP 29-32.

3. Gorshkov A.S. 3D Graphics Algorithms Package for CAD-CAM Applications. SAPR i Graphika. N 3. 2003.

4. The Universal Method of Seismic Cube Visualization and Processing Based on Fourier-Spline Decomposition. / Gorshkov A.S. // Abstracts for «Geomodel-2008».-22-25/9-2008

5. The Universal System for Seismic Cube Isosurfaces Synthesis. / Gorshkov A.S. // Abstracts for «Geomodel -2009».-7-10/9-2009.

6. The Universal Algorithm for Parametric Maps with Faults Synthesis Based on FourierSplines. / Gorshkov A.S. // Abstracts for «Geomodel 2016».-11-15/9-2016. 